\title{
Consumo de carnes por adultos do sul do Brasil: um estudo de base populacional
}

\author{
Meat consumption by adults in southern Brazil: \\ a population-based study
}

Bruna Celestino Schneider ${ }^{1}$

Suele Manjourany Silva Duro ${ }^{1}$

Maria Cecília Formoso Assunção ${ }^{1}$

${ }^{1}$ Programa de Pós-

Graduação em

Epidemiologia,

Universidade Federal

de Pelotas. R. Marechal

Deodoro $1160 / 3^{\circ}$ Piso,

Centro. 96.020-220 Pelotas

RS Brasil.

brucelsch@yahoo.com.br
Abstract This paper is the result of a cross-sectional population-based study that evaluated individuals aged 20 or more living in the urban area of Pelotas in the state of Rio Grande do Sul and sought to describe the frequency of meat consumption and the habit of eating meat with an excess of fat. It evaluated the consumption over the past year of red meat (including bone, steak and ground beef), white meats (chicken and fish) and viscera and processed meats. Of the 2,730 people interviewed, $99.1 \%\left(\mathrm{CI}_{95 \%} 98.7\right.$ - 99.5) consumed some type of meat over the past year, while around $32 \%$ reported daily consumption. The prevalence of consumption of red meat ( $99.3 \% \mathrm{CI}_{95 \%} 98.9$ 99.6) and white meat (99.4\% $C_{95 \%} 99.1$ - 99.7) was similar. Chicken was the meat most consumed by interviewees (98.0\% CI ${ }_{95 \%} 97.4$ - 98.5), while viscera were the least consumed ( $59.1 \% \mathrm{CI}_{950 \%} 56.4$ - 61.7). The processed meats consumed by $85.5 \%$ $\left(C I_{95 \%} 83.7-87.2\right)$ of the respondents, presented the highest prevalence of daily consumption (16.6\%). Meats with excess of fat were consumed by $52.3 \%$ $\left(C_{95 \%} 49.8\right.$ - 54.8) of adults, especially men and people of lower education and economic status.

Key words Food consumption, Meat, Adult, Cross-sectional studies
Resumo Estudo transversal de base populacional que avaliou indivíduos com 20 anos ou mais, residentes na zona urbana de Pelotas, Rio Grande do Sul, que objetivou descrever a frequência do consumo de carnes e o hábito de consumi-las com excesso de gordura. Foi avaliado, no último ano, o consumo de carnes vermelhas (com osso, bife e carne moída), brancas (frango e peixes), vísceras e embutidos. Dos 2,730 entrevistados, $99,1 \%$ ( $I C_{95 \%} 98,7$ - 99,5) consumiu algum tipo de carne no último ano, sendo que, em torno de $32 \%$ referiu consumo diário. A prevalência do consumo de carnes vermethas $\left(99,3 \%, I C_{95 \%}, 98,9\right.$ - 99,6) e brancas $(99,4 \%$ $I C_{95 \%}, 99,1$ - 99,7) foi semelhante. A carne de frango foi a mais consumida $\left(98,0 \%, I C_{95 \%}, 97,4-98,5\right)$, enquanto que as vísceras, as menos $\left(59,1 \%\right.$ IC $_{95 \%}$ 56,4 - 61,7). Os embutidos, consumidos por $85,5 \%$ $\left(I_{95 \%}, 83,7\right.$ - 87,2) das pessoas, apresentaram a maior prevalência de consumo diário (16,6\%). As carnes com excesso de gordura foram consumidas por $52,3 \%\left(\mathrm{IC}_{95 \%}, 49,8-54,8\right)$ dos adultos, principalmente homens, e pessoas de menor escolaridade e nivel econômico.

Palavras-chave Consumo de alimentos, Carne, Adulto, Estudos transversais 


\section{Introdução}

A dieta equilibrada é um requisito fundamental e indispensável para a qualidade de vida e longevidade das populações. A alimentação é um dos fatores de risco ou proteção modificável que pode ser desencorajado ou incorporado à vida das pessoas. E é sabido que modificações no perfil alimentar resultam em mudanças nos padrões de morbimortalidade ${ }^{1}$.

A carne é um alimento de origem animal, fonte de proteínas de alto valor biológico e rica em vitaminas do complexo $B$ e em minerais de alta biodisponibilidade como ferro e zinco ${ }^{2}$. Os peixes, principalmente das espécies arenque, anchova, salmão, sardinha e truta, têm alta concentração de ácidos graxos poli-insaturados essenciais, da série ômega 3 , os quais estão relacionados à prevenção de doenças cardiovasculares ${ }^{3}$. Além disso, são nutricionalmente ricos em cálcio, vitaminas $\mathrm{A}$, E e D, devendo o seu consumo ser encorajado ${ }^{3,4}$.

No Brasil, a contribuição calórica das carnes na dieta vem sofrendo uma evolução crescente. Aumentou de 9,0\% em 1975 para 13,1\% em 20035. Por outro lado, o grupo das carnes é o item mais caro da alimentação. Segundo a Pesquisa de Orçamento Familiar (POF), de $2008-09^{6}$, as carnes, vísceras e pescados representaram $15,1 \%$ dos gastos com alimentação. E apesar deste percentual estar diminuindo nos últimos anos, visto que na POF $2002-03^{7}$ correspondia a $18,3 \%$, o gasto ainda é alto em relação a outros alimentos.

Embora a carne seja um item que deva estar presente em uma dieta equilibrada, o alto consumo deste alimento, principalmente se contiver excesso de gordura, tem sido apontado como um fator preocupante. As carnes, especialmente as vermelhas e as processadas [também conhecidas como embutidos (salsichas, salames, presuntos e etc.)], possuem alta concentração de colesterol, ácidos graxos saturados e sódio ${ }^{2,3}$, estando assim associadas à incidência de doenças cardiovasculares e até mesmo de câncer-10.

Particularmente no sul do Brasil, em Porto Alegre, a Vigilância de Fatores de Risco e Proteção para Doenças Crônicas por Inquérito Telefônico (Vigitel) mostrou que, apesar de ter ocorrido uma discreta diminuição no consumo de carnes com excesso de gordura entre adultos $(40 \% \mathrm{em}$ $2006^{11}, 34,8 \%$ em $2010^{12}$ ), ainda é observada uma alta frequência.

No intuito de promover hábitos saudáveis de alimentação, o Ministério da Saúde (MS) em 2006, com base nas recomendações da Organização Mundial da Saúde ${ }^{13}$, desenvolveu um guia alimentar para a população brasileira ${ }^{14}$, orientando o consumo diário de uma porção de carne com a retirada da gordura aparente antes da preparação. O guia também salienta que se deve dar preferência às carnes brancas e que as vísceras devem ser consumidas uma vez na semana, enquanto que as carnes processadas somente ocasionalmente.

Dessa forma, descrever o consumo de carnes, principalmente na cidade de Pelotas/RS, que possui expressivo rebanho bovino de corte e é dotada de uma lagoa com grande potencial para produção de pescado ${ }^{15}$, assim como identificar o hábito de consumir carnes com excesso de gordura, poderá subsidiar o direcionamento de ações visando à promoção de uma alimentação mais saudável em termos locais e regionais.

\section{Métodos}

Pelotas é um município de médio porte localizado no sul do estado do Rio Grande do Sul (RS), contando com aproximadamente 350 mil habitantes, sendo $90 \%$ destes residentes na zona urbana ${ }^{16}$. Um estudo transversal de base populacional, em adultos com 20 anos ou mais, foi realizado na zona urbana de Pelotas, durante o período de janeiro a junho de 2010, com o objetivo de conhecer características da saúde da população. Esse trabalho foi realizado no Programa de Pós-Graduação em Epidemiologia da Universidade Federal de Pelotas, através de esforço conjunto que culminou na realização de um trabalho de campo único e denominado consórcio de pesquisa ${ }^{17}$, no qual todos os mestrandos participaram de maneira integral e do qual puderam obter dados para suas dissertações.

\section{Tamanho de amostra}

Para determinar o número necessário de pessoas que seriam estudadas quanto a frequência de consumo de carnes, utilizou-se para o cálculo do tamanho da amostra parâmetros de consumo semanal de peixes no último ano, entre adultos de 23 anos pertencentes à Coorte de Nascimentos de Pelotas (RS), 1982, que foi de $18 \%$. Considerando um erro aceitável de três pontos percentuais, calculou-se como necessário estudar 1.382 indivíduos. Para verificar as associações do consumo de carnes com características demográficas e socioeconômicas, considerando uma significância de 5\%, nível de confiança de $95 \%$, poder de $80 \%$ para detectar razões de prevalências de no mínimo 2,0, levando em consideração um efeito de delineamento de 2,0 e acrescentando-se 10\% para suprir eventuais 
perdas e recusas e 15\% para controle de possíveis fatores de confusão, calculou-se como necessário estudar 3.638 indivíduos. Este foi o maior tamanho amostral necessário, visto que, para a variável idade, foi considerada uma prevalência de exposição de $10 \%$ na faixa de 40 a 59 anos.

\section{Amostragem}

Inicialmente, verificou-se o número de domicílios necessários para atender os objetivos de todos os integrantes da pesquisa, o qual foi de 1.300. Com o objetivo de minimizar o efeito de delineamento (DEF), oriundo da amostragem por conglomerados, optou-se por trabalhar com mais setores censitários e menor número de domicílios em cada setor ( 130 setores censitários e cerca de 10 domicílios por setor). Dessa forma, ordenaram-se os 404 setores censitários domiciliares da cidade de Pelotas ${ }^{16}$, conforme a ordem crescente de renda média mensal do chefe da família. Em seguida, somou-se o número de domicílios do primeiro ao último setor (92.407) e dividiu-se pelo número de setores censitários previstos (130), de forma a se obter um número que seria o "pulo" para a seleção sistemática dos setores censitários. Um número entre um e o "pulo" foi selecionado aleatoriamente e assim obteve-se o primeiro domicilio, o qual constava no primeiro setor a ser incluído na amostra. Após, somou-se o valor do "pulo" ao número selecionado para identificar o próximo setor censitário da amostra. Este processo foi repetido até que 130 setores fossem selecionados.

Posteriormente, os 130 setores foram visitados para atualização do número de domicílios, já que o último censo demográfico foi realizado há 10 anos $^{16}$. Para manter a equiprobabilidade, ao detectar-se diferença entre o número de domicílios encontrados no setor censitário e o número referido pelo último censo, estes foram contabilizados e participaram da seleção sistemática da qual resultou o número de domicílios elegíveis para o estudo.

A seleção de domicílios dentro de cada setor censitário obedeceu a uma lógica semelhante à descrita anteriormente. O número de domicílios de cada setor foi dividido por 10, e assim era obtido o "pulo". Um número entre um e o "pulo" de cada setor foi determinado de forma aleatória, indicando o primeiro domicílio a ser visitado. Os outros domicílios foram selecionados pela soma do valor do "pulo" ao último domicílio, repetindo este processo até o fim do setor censitário. Desta forma, foram eleitos 1.512 domicílios para a pesquisa.

\section{Variáveis dependentes}

Foi avaliada a frequência do consumo de diferentes carnes por indivíduos com 20 anos ou mais, residentes na zona urbana de Pelotas (RS). Para tal, foi utilizado um questionário de frequência alimentar (QFA) qualitativo, com período recordatório de 12 meses. Investigou-se sobre o consumo de diferentes tipos de carne, bem como a frequência de consumo de cada tipo: vermelha com osso (costela, agulha ou paleta), vermelha sem osso (bife ou pedaços), vermelha sem osso (carne moída ou guisado), frango ou galinha, peixes frescos ou enlatados (sardinha e atum) ou camarão, embutidos (linguiça, salsicha, presunto, mortadela, salame...) e vísceras ou miúdos (coração, fígado, moela...).

O hábito de consumir carnes com excesso de gordura foi investigado a partir do questionamento: $O$ (a) Sr. (a) tira a gordura da carne vermelha antes de comer? e $\mathrm{O}$ (a) Sr. (a) tira a pele do frango antes de comer? Os embutidos, apesar de serem carnes com excesso de gordura, não foram considerados nessa categoria, para fins de comparação com dados nacionais, os quais se baseiam na gordura visível das carnes.

As informações sobre a frequência de consumo das diferentes carnes foram categorizadas em: "menos de 1 vez por semana", "1 a 3 vezes por semana", "4 a 6 vezes por semana" e "diário". As variáveis "retira a gordura aparente da carne vermelha antes de comer" e "retira a pele do frango antes de comer" foram agrupadas e formaram uma variável dicotômica denominada: "consumo de carnes com excesso de gordura”. Considerou-se como consumidor de carnes gordurosas, aqueles indivíduos que consumiam ao menos uma das carnes com gordura anteriormente citadas.

\section{Variáveis independentes}

Para caracterizar os consumidores de carnes, avaliou-se: o sexo e cor da pele (branca e não branca), que foram objeto de observação pelas entrevistadoras; idade, coletada como variável numérica discreta e posteriormente categorizada em 20 a 29 anos, 30 a 39 anos, 40 a 49 anos, 50 a 59 anos, 60 anos ou mais; escolaridade, registrada como variável numérica discreta e depois categorizada em 0 a 4 anos, 5 a 8 anos, 9 a 11 anos, 12 ou mais; e o nível econômico, coletado segundo instrumento proposto pela Associação Brasileira de Empresas de Pesquisas (Abep) ${ }^{18}$ e categorizado em A/B (mais alto), C e D/E (mais baixo), que foram referidos pelos entrevistados. 


\section{Coleta de dados}

Os dados foram coletados de janeiro a maio de 2010, por entrevistadoras treinadas e através de um questionário previamente testado e construído em formato digital que foi preenchido na tecnologia de Personal Digital Assistents (PDA).

Para minimizar as perdas, as entrevistadoras visitavam os domicílios pelo menos três vezes em dias e horários diferentes e, caso ainda persistisse a falta de contato, a mesma comunicava o seu supervisor, que fazia tentativas para reverter a situação. Quando o indivíduo recusava-se a participar do estudo, a entrevistadora comunicava seu supervisor, que igualmente tentava reverter a situação.

\section{Controle de qualidade}

A qualidade das informações obtidas foi garantida através de reuniões semanais das entrevistadoras com seus supervisores, para esclarecimento de dúvidas, entrega e checagem dos dados coletados. Além dessas medidas, foram refeitas pelos supervisores do estudo $11 \%$ das entrevistas de cada setor censitário, escolhidas aleatoriamente através de um questionário padronizado e simplificado. Para este estudo, a questão utilizada foi: $O(a) S r$. (a) tira a pele do frango antes de comer?. Foi testada a concordância esperada, além do acaso entre as respostas através do cálculo do Kappa, o qual resultou em $87,2 \%$ de repetibilidade.

\section{Análise de dados}

Os dados provenientes dos PDAs foram transferidos para um banco de dados único em formato Excel. Após análise de consistência, os mesmos foram transferidos para o programa Stata, versão 11.0, no qual, inicialmente, foi realizada uma análise descritiva para caracterização da população estudada e o cálculo das prevalências de consumo dos diferentes tipos de carnes. As análises bivariadas consistiram de testes Qui-quadrado de Pearson, com cálculo do valor p para heterogeneidade nas exposições categóricas nominais, assim como o valor p para tendência linear nas exposições ordinais. O DEF foi considerado em todas as análises que foram realizadas utilizando o comando svy.

\section{Ética}

O protocolo do presente estudo foi submetido e aprovado pela Comissão de Ética em Pesquisa da aceitaram participar do estudo assinaram um Termo de Consentimento Livre e Esclarecido (TCLE).

\section{Resultados}

Nos 130 setores censitários sorteados, foram encontrados 3.059 indivíduos elegíveis para o estudo. Foram contabilizadas 327 perdas e recusas $(10,7 \%)$, totalizando 2.732 adultos entrevistados. Para o presente estudo, foram excluídas duas pessoas que se alimentavam através de sonda.

Foi calculado o DEF para todas as variáveis dependentes do presente estudo. O maior valor encontrado foi para o consumo de peixes $(3,19)$ e o menor para o consumo de frango $(1,06)$.

Dos indivíduos entrevistados, em torno de $60 \%$ era do sexo feminino e mais de $80 \%$ de cor de pele branca. A faixa etária de adultos jovens (20 a 29), bem como de 60 anos ou mais, correspondeu a $21,8 \%$ e $23,2 \%$, respectivamente. Aproximadamente $30 \%$ dos indivíduos relatou ter estudado entre cinco e oito anos, e cerca de $50 \%$ pertencia ao nível econômico $\mathrm{C}$.

O consumo de algum tipo de carne no último ano foi referido por 99,1\% ( $\mathrm{IC}_{95 \%}, 98,7$ - 99,5) dos entrevistados, sendo que aproximadamente 32\% consumiam diariamente esse alimento, não se evidenciando diferença entre homens e mulheres.

A carne mais consumida pelos pelotenses entrevistados foi a carne de frango $\left(98,0 \%, \mathrm{IC}_{95 \%}, 97,4\right.$ - 98,5) e a menor prevalência de consumo foi de vísceras $\left(59,1 \%, \mathrm{IC}_{95 \%} 56,4-61,7\right)$. Os embutidos foram referidos por $85,5 \%\left(\mathrm{IC}_{95 \%}, 83,7-87,2\right)$ dos indivíduos. O grupo das carnes vermelhas, composto pelas carnes com osso, bife e carne moída, foi consumido por 99,3\% ( $\left.\mathrm{IC}_{95 \%}, 98,9-99,6\right)$ das pessoas no último ano, sendo o maior consumo de carnes com osso $\left(93,0 \% \mathrm{IC}_{95 \%} 91,9\right.$ - 94,2) seguida da carne moída $\left(91,8 \%\right.$, IC $_{95 \%}, 90,5$ - 93,1) e do bife $\left(87,6 \%, \mathrm{IC}_{95 \%}, 85,6\right.$ - 89,5). Estas carnes foram discretamente mais consumidas por homens. O consumo de carnes brancas (frango e peixes) também foi expressivo, 99,4\% ( $\mathrm{IC}_{95 \%}$, 99,1 - 99,7), sendo preeminente o consumo de frango. $\mathrm{O}$ consumo de peixes foi referido por $81,8 \%\left(\mathrm{IC}_{95 \%}\right.$, 79,2 - 84,4) dos adultos entrevistados.

A frequência de consumo mais referida entre as diferentes carnes foi de " 1 a 3 vezes por semana", conforme mostra a Tabela 1 , sendo a carne de frango $\left(75,4 \%, \mathrm{IC}_{95 \%} 97,4-98,5\right)$ e a carne moída $\left(72,9 \%, \mathrm{IC}_{95 \%}, 70,5-75,3\right)$ as mais consumidas desta categoria. As vísceras e os peixes foram as carnes que apresentaram a menor frequência de consumo, uma vez que a maioria dos indivíduos consumiu peixes $\left(66,6 \%, \mathrm{IC}_{95 \%}, 63,8-69,5\right)$ e vísceras $\left(71,3 \%, \mathrm{IC}_{95 \%}, 68,5\right.$ - 74,1) menos de uma vez por semana. Em relação ao consumo diário de carnes, notou-se um predomínio dos embutidos 
Tabela 1. Consumo de carnes segundo características sócio demográficas e frequência de consumo pelos adultos entrevistados. Pelotas/RS, 2010.

\begin{tabular}{|c|c|c|c|c|c|}
\hline \multirow[b]{2}{*}{ Variáveis } & \multirow[b]{2}{*}{$\begin{array}{c}\text { Carnes vermelhas } \\
\%(\mathrm{IC} 95 \%)\end{array}$} & \multicolumn{2}{|c|}{ Carnes brancas } & \multirow[b]{2}{*}{$\begin{array}{l}\text { Embutidos } \\
\%(\text { IC95\%) }\end{array}$} & \multirow[b]{2}{*}{$\begin{array}{l}\text { Vísceras } \\
\%(I C 95 \%)\end{array}$} \\
\hline & & $\begin{array}{c}\text { Frango } \\
\%(\text { IC95\%) }\end{array}$ & $\begin{array}{c}\text { Peixes } \\
\%(\text { IC95\%) }\end{array}$ & & \\
\hline Sexo & $0,04^{*}$ & $0,02^{*}$ & $0,1^{*}$ & $<0,0001^{*}$ & $0,01^{*}$ \\
\hline Masculino & $99,7(99,3-99,9)$ & $97,3(96,3-98,2)$ & $83,0(79,9-86,2)$ & $89,0(86,9-91,2)$ & $62,0(58,4-65,7)$ \\
\hline Feminino & $99,0(98,4-99,5)$ & $98,5(97,9-99,1)$ & $80,9(78,1-83,6)$ & $82,9(80,8-85,0)$ & $56,9(54,0-59,8)$ \\
\hline Cor da Pele & $0,9^{*}$ & $0,6^{*}$ & $0,1^{*}$ & $0,5^{*}$ & $0,6^{*}$ \\
\hline Branca & $99,3(98,9-99,6)$ & $98,0(97,4-98,7)$ & $82,4(79,6-85,1)$ & $85,3(83,4-87,1)$ & $59,3(56,4-62,2)$ \\
\hline Não branca & $99,2(98,3-100)$ & $97,6(96,2-99,1)$ & $79,3(75,0-83,6)$ & $86,4(82,9-90,0)$ & $57,8(52,6-62,9)$ \\
\hline Idade (anos) & $0,03^{*}$ & $0,2^{*}$ & $0,001^{*}$ & $<0,001^{* *}$ & $<0,001^{* *}$ \\
\hline 20 a 29 & $99,7(99,1-100)$ & $98,5(97,5-99,4)$ & $77,7(73,8-81,6)$ & $94,1(92,3-95,9)$ & $64,2(60,3-68,0)$ \\
\hline 30 a 39 & $99,6(99,0-100)$ & $99,1(98,3-99,9)$ & $85,3(81,5-89,1)$ & $92,3(89,4-95,3)$ & $62,7(58,0-67,4)$ \\
\hline 40 a 49 & $99,4(98,8-100)$ & $97,2(95,7-98,7)$ & $84,2(80,5-87,9)$ & $85,3(81,9-88,7)$ & $59,9(54,9-64,8)$ \\
\hline 50 a 59 & $99,0(98,1-99,7)$ & $97,6(96,0-99,1)$ & $84,4(80,6-88,2)$ & $84,2(81,0-87,4)$ & $60,5(55,6-65,3)$ \\
\hline 60 ou mais & $98,7(97,8-99,7)$ & $97,6(96,4-98,8)$ & $79,0(74,8-83,1)$ & $73,5(69,4-77,7)$ & $49,8(45,9-53,6)$ \\
\hline Escolaridade (anos) & $0,07^{* *}$ & $0,8^{*}$ & $<0,001^{* *}$ & $<0,001^{* *}$ & $<0,001^{* *}$ \\
\hline 0 a 4 & $98,6(97,6-99,6)$ & $98,4(97,3-99,5)$ & $77,0(72,0-81,9)$ & $76,6(73,3-80,9)$ & $49,4(44,5-54,3)$ \\
\hline 5 a 8 & $99,6(99,2-100)$ & $97,8(96,7-98,9)$ & $78,0(73,9-82,1)$ & $83,9(81,2-86,5)$ & $59,6(55,4-63,8)$ \\
\hline 9 a 11 & $99,6(99,1-100)$ & $97,9(96,9-99,0)$ & $87,0(84,0-89,9)$ & $92,2(90,2-94,2)$ & $62,1(58,3-66,0)$ \\
\hline 12 ou mais & $99,6(99,1-100)$ & $98,3(97,2-99,4)$ & $88,9(85,7-92,0)$ & $92,0(89,5-94,5)$ & $67,5(63,1-72,0)$ \\
\hline Nível Econômico & $0,2^{*}$ & $0,8^{*}$ & $<0,001^{* *}$ & $<0,001^{* *}$ & $<0,001^{\star *}$ \\
\hline A/B (alto) & $99,8(98,2-99,6)$ & $97,7(96,0-99,4)$ & $91,3(88,4-94,1)$ & $91,1(88,4-93,7)$ & $67,6(62,5-72,8)$ \\
\hline $\mathrm{C}$ & $99,3(98,9-99,8)$ & $97,9(97,2-98,7)$ & $84,4(81,7-87,2)$ & $86,6(84,7-88,5)$ & $61,5(58,0-64,9)$ \\
\hline D/E (baixo) & $98,9(99,3-100)$ & $98,2(97,3-99,0)$ & $73,3(69,4-77,2)$ & $81,0(77,7-84,0)$ & $51,4(47,2-55,6)$ \\
\hline Frequência de consumo & - & - & - & - & - \\
\hline$<1$ vez & $2,5(1,8-3,1)$ & $8,8(7,5-10,1)$ & $66,6(63,8-69,5)$ & $20,3(18,1-22,4)$ & $71,3(68,5-74,1)$ \\
\hline 1 a 3 vezes & $61,0(58,5-63,4)$ & $75,4(73,3-77,5)$ & $28,5(25,9-31,2)$ & $53,1(50,8-55,4)$ & $23,5(20,9-26,0)$ \\
\hline 4 a 6 vezes & $21,8(20,0-23,6)$ & $10,7(9,2-12,2)$ & $0,9(0,4-1,3)$ & $10,1(8,7-11,5)$ & $0,8(0,2-1,4)$ \\
\hline Diário & $14,8(12,7-16,9)$ & $5,1(3,9-6,3)$ & $4,0(3,0-5,0)$ & $16,6(14,6-18,5)$ & $4,4(3,3-5,5)$ \\
\hline
\end{tabular}

"Teste Qui-quadrado para heterogeneidade. * Teste Qui-quadrado para tendência linear.

$\left(16,6 \%, \mathrm{IC}_{95 \%,} 14,6\right.$ - 18,5) e das carnes vermelhas $\left(14,8 \%, \mathrm{IC}_{95 \%,} 12,7-16,9\right)$.

Na Tabela 1 também é apresentada a relação entre as características demográficas e socioeconômicas dos entrevistados e o consumo das diferentes carnes avaliadas. Notou-se que o consumo de peixes, embutidos e vísceras foi mais frequente entre os homens. Já a carne de frango foi mais frequentemente consumida pelas mulheres. Em relação à idade, observou-se um menor consumo de peixes pelos adultos de 20 a 29 anos $(77,7 \%$,

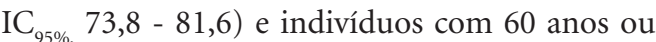
mais $\left(79,0 \%, \mathrm{IC}_{95 \%} 74,8\right.$ - 83,1). Para os embutidos e vísceras evidenciou-se uma diminuição na prevalência de consumo à medida que a idade aumenta. A escolaridade e o nível econômico dos adultos avaliados apresentaram uma relação direta com o consumo de peixes, embutidos e vísceras.

As Figuras 1, 2, 3 e 4 mostram, respectivamente, a relação entre a frequência de consumo das carnes vermelhas, frango, peixes e embutidos com o nível econômico dos entrevistados. Destaca-se que, com exceção dos embutidos, as carnes vermelhas e brancas apresentaram uma maior prevalência de consumo diário pelos indivíduos do menor nível econômico (carnes vermelhas $16,2 \%$; frango - $8,7 \%$; peixes $-6,4 \%$ ) ao mesmo tempo em que indivíduos do menor nível econômico referiram mais frequentemente o consumo na categoria "menos de 1 vez por semana" (carnes vermelhas - 4,1\%; frango - 9,2\%; peixes - 67,7\%). Em relação aos embutidos, observou-se que os indivíduos do nível econômico mais alto consumiram diariamente esse alimento em maior proporção (22,0\%), comparados àqueles do nível econômico mais baixo, entre os quais foi observada uma maior prevalência na categoria "menos de 1 vez por semana" $(22,8 \%)$.

O consumo de carnes com excesso de gordura foi referido por 52,3\% $\left(\mathrm{IC}_{95 \%} 49,8-54,8\right)$ dos 


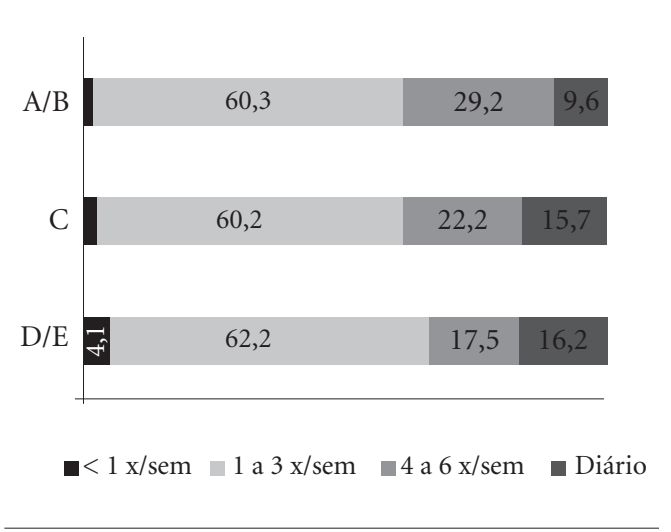

Figura 1. Frequência do consumo de carnes vermelhas conforme nível econômico dos adultos avaliados. Pelotas/RS, 2010. (Valor < 5\% não apresentado)

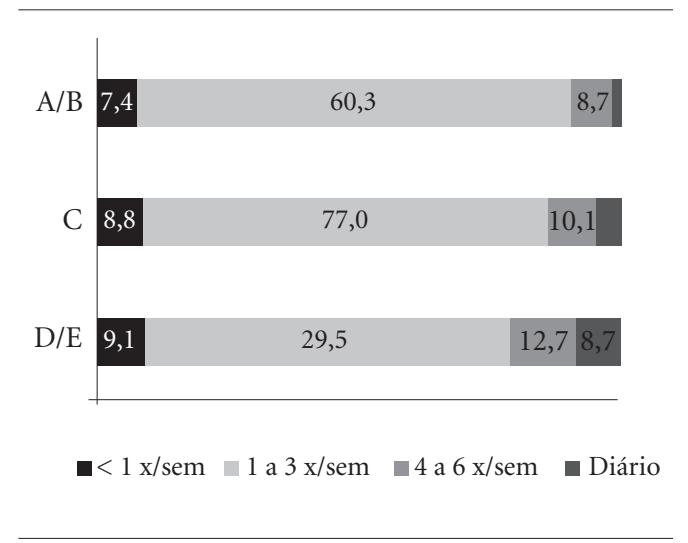

Figura 2. Frequência do consumo de frango conforme nível econômico dos adultos avaliados. Pelotas/RS, 2010. (Valor $<5 \%$ não apresentado)

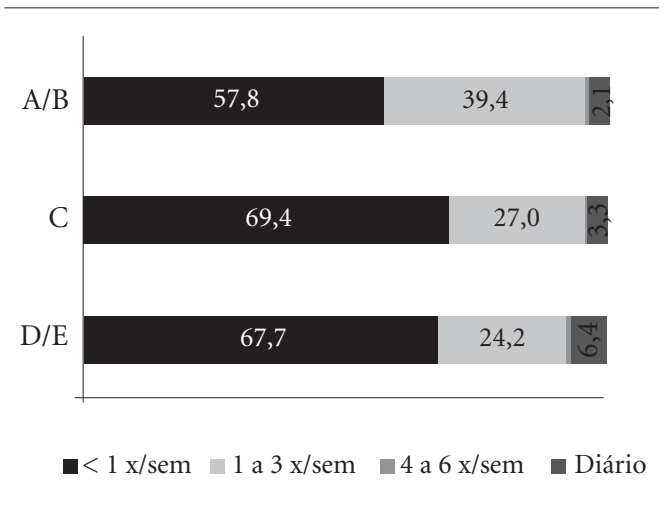

Figura 3. Frequência do consumo de peixes conforme nível econômico dos adultos avaliados. Pelotas/RS, 2010. (Valor $<5 \%$ não apresentado)

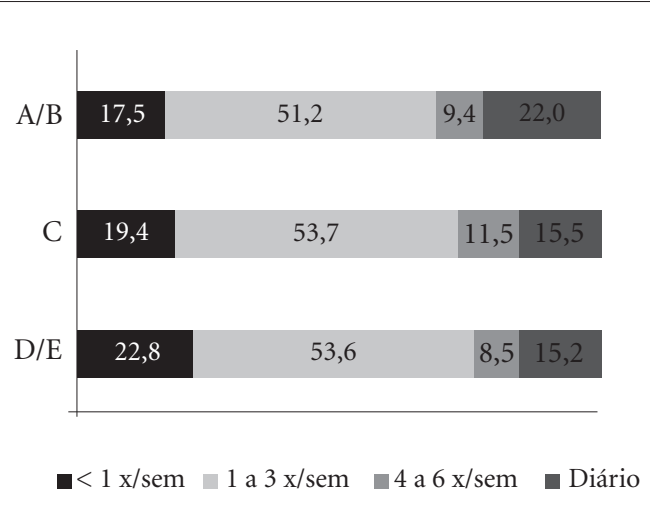

Figura 4. Frequência do consumo de embutidos conforme nível econômico dos adultos avaliados. Pelotas/RS, 2010.

indivíduos, sendo $66,7 \%\left(\mathrm{IC}_{95 \%}, 63,6\right.$ - 69,8) entre os homens e $41,8 \%\left(\mathrm{IC}_{95 \%}, 39,2-44,5\right)$ entre as mulheres. Os entrevistados que apresentaram mais frequentemente esse hábito foram aqueles com idade entre 30 e 39 anos, com escolaridade igual ou menor que oito anos, e aqueles com nível econômico mais baixo (D/E). Ao estratificar por sexo, observou-se que os homens têm um consumo cerca de $60 \%$ maior que as mulheres em relação a todas as variáveis de exposição analisadas (dados não apresentados).

\section{Discussão}

O consumo de carnes é um tema que pouco tem sido abordado na literatura. Alguns estudos internacionais relacionam esse hábito com o surgimento de doenças, mas poucas são as pesquisas que tratam do consumo desse alimento. As informações trazidas pelo presente artigo são importantes para que se possa conhecer alguns hábitos alimentares da população, já que este é o primeiro estudo de base populacional na região que descreve a frequência do consumo de diferentes tipos de carne e a ingestão destas com excesso de gordura.

O instrumento de coleta de dados "QFA" foi qualitativo não medindo, portanto, a porção de carne ingerida pelos entrevistados o que dificultou comparações com as recomendações do $\mathrm{MS}^{14} \mathrm{e}$ alguns estudos. Cabe ressaltar que não foi possível discriminar os tipos de peixes ingeridos pelos indivíduos devido ao fato que de estudos prévios para testar o questionário apontarem um baixo consumo. 
Esta pesquisa atestou que 99,1\% da população avaliada consumiu algum tipo de carne no último ano. Segundo a Food and Agriculture Organization $(\mathrm{FAO})^{2}$, o consumo de carnes está intimamente relacionado à disponibilidade do alimento, preço, tradição, economia, influências religiosas e até mesmo à geografia do local. O Brasil revelou um aumento de $50 \%$ na participação relativa das carnes, no total de calorias da dieta da população desde a década de 70 até $2003^{5}$ e está entre os países em desenvolvimento aonde mais vem crescendo a produção de carnes ${ }^{19}$. Entre os maiores produtores mundiais estão Argentina e Uruguai ${ }^{20}$, países próximos do estado do RS em termos de localização. Portanto, o alto consumo de carnes apresentado nesta pesquisa pode estar ligado a estes dois fatores somados a extensa produtividade pecuarista do RS, principalmente de carne suína e ovina ${ }^{21}$ e à produção de pescado e rebanho bovino de corte da cidade de Pelotas ${ }^{15}$.

No Distrito Federal, um estudo transversal com indivíduos de 15 anos ou mais, mostrou uma prevalência de consumo de $87,8 \%$ de carne bovina, $91,4 \%$ para frango e $33,6 \%$ de peixes $^{22}$. No Rio de Janeiro, constataram que, entre os adultos entrevistados, em torno de $97 \%$ dos homens e $94 \%$ das mulheres consumiam carne de boi ${ }^{23}$. Pesquisas internacionais também descrevem o consumo de carnes. Nos Estados Unidos, dados do Coronary Artery Risk Development in Young Adults Study, mostraram que menos de $1 \%$ dos 5.111 adultos avaliados não consumiam carne ${ }^{24}$. $\mathrm{Na}$ Irlanda, $88 \%$ dos adultos entrevistados consumia carnes vermelhas e $79 \%$ carnes brancas e embutidos ${ }^{25}$.

Devido à importância nutricional da carne, o MS, através do Guia Alimentar para a População Brasileira ${ }^{14}$, recomenda o consumo diário de uma porção. Este estudo mostrou que apesar do alto relato de consumo de carnes, menos de 1/3 dos entrevistados as consumiam diariamente. A frequência do consumo é um fator complexo, já que envolve a preferência alimentar dos indivíduos, o hábito, a cultura, o modismo e principalmente o custo. A carne é um alimento dispendioso para os brasileiros. Segundo dados recentes da POF 2008$09^{6}, 15,1 \%$ do gasto das famílias com alimentação é oriundo da carne, vísceras e pescados, e o grupo de menor renda é o que proporcionalmente mais gasta com a compra desse gênero alimentício. Levy-Costa et al. $^{5}$ apontaram para uma relação direta entre a participação relativa das carnes nas calorias da dieta dos brasileiros com o nível de rendimentos familiares.

$\mathrm{Na}$ presente pesquisa, o frango foi a carne branca mais consumida e cerca de $90 \%$ das pessoas referiram um consumo mínimo de uma vez por semana. Um estudo de série temporal com adultos brasileiros ${ }^{26}$ notou um aumento no consumo de carnes na década de 70, devido a um crescimento frequente no consumo de frango. Concordando, Levy-Costa et al. ${ }^{5}$ referiram um incremento de $100 \%$ na participação relativa desta carne nas calorias totais da dieta de 1974 a 2003. O Ministério da Agricultura mostra que a produção de frango no Brasil em 2006 sofreu um acréscimo de quatro vezes a produção na década de $90^{21}$. Portanto, o alto consumo de frango pelos pelotenses pode ser explicado pela crescente produção de aves no país, que levou à diminuição do custo deste alimento e o tornou mais acessível para o consumo.

No entanto, o consumo de peixes no último ano, apesar de ter sido apontado por $82 \%$ dos indivíduos, foi bem menos frequente. Cerca de $30 \%$ das pessoas referiram consumir peixes pelo menos uma vez na semana. Esse tipo de carne é referenciado por seu alto conteúdo de ácidos graxos poliinsaturados essenciais, da série ômega $3^{2}$. O ômega 3 é reconhecido na literatura pelo seu potencial na redução do risco de desenvolvimento de doenças cardiovasculares e diminuição dos níveis lipídicos sanguíneos ${ }^{3,27}$ e, embora nem todos os peixes possuam quantidades significativas deste ácido graxo, esta carne é considerada a mais saudável para o consumo, uma vez que não vem sendo associada a desfechos maléficos para a saúde ${ }^{4}$. O consumo pouco frequente de peixes observado neste trabalho, vai ao encontro da evolução da participação relativa desse alimento no total de calorias da dieta dos adultos brasileiros, que sofreu uma diminuição de cerca de 50\% de 1974 até $2003^{5}$. Porém, especificamente em Pelotas, onde se tem uma colonina de pescadores que produz pescado a partir da Lagoa dos Patos, o fato de as pessoas consumirem peixes com pouca frequência não está ligado à disponibilidade desse alimento, mas sim ao acesso. $\mathrm{O}$ alto custo dos peixes, talvez justificado por problemas relacionados à política pesqueira, não supera sequer a época de maior produção na região, geralmente em meados do verão e início do outono, porque ainda assim, apesar do custo relativamente diminuir, os indivíduos ainda consomem esse tipo de carne com pouca frequência. Esta pesquisa examinou o percentual de pessoas e o consumo na época, detectando que apenas 5,3\% consomem peixes pelo menos uma vez por semana na época de maior produção de pescado, e não o fazem no restante do ano.

Ficou evidenciada na presente pesquisa a relação entre o nível econômico dos indivíduos e a frequência de consumo de carnes. Pessoas de nível 
econômico mais baixo apresentaram um consumo diário de carnes vermelhas 60\% maior em relação aos do nível mais alto. Entretanto, no que tange à qualidade das carnes em termos de gorduras saturadas e colesterol, verifica-se que integrantes do nível econômico mais baixo consumiram com frequência cinco vezes maior as carnes vermelhas com osso e sete vezes mais a carne moída, que os indivíduos do nível alto. As carnes vermelhas com osso e moída podem apresentar maior concentração de gorduras saturadas em relação ao bife ${ }^{2}$. A POF 2008-096 mostrou que os brasileiros com os menores rendimentos mensais gastavam mais com carne de segunda do que os indivíduos de melhores rendimentos. Este fato gera uma preocupação sobre o maior risco de doenças ocasionadas pelo consumo excessivo de gorduras no grupo de mais baixa renda.

Este trabalho detectou também que os embutidos foram consumidos pelo menos uma vez na semana por cerca de $80 \%$ dos entrevistados, sendo que quase $17 \%$ destes referiram um consumo diário. Os homens consumiram mais em relação às mulheres, assim como ocorreu também com relação às pessoas de melhores condições econômicas e escolaridade. No Brasil, houve um aumento de $300 \%$ na participação destas carnes na dieta dos anos 70 a $2003^{5}$. Os embutidos contêm níveis significativos de agentes cancerígenos, os nitritos e nitratos, além de grande quantidade de gorduras saturadas, colesterol e sódio ${ }^{2,10}$, e é sabido que o consumo contínuo de alimentos gordurosos vem sendo associado ao aumento no risco de câncer e doenças cardíacas, portanto, o MS recomenda que se modere ou até mesmo evite seu consumo ${ }^{10,14}$.

O consumo de carnes com excesso de gordura, avaliado pelo o consumo da pele do frango e/ou gordura aparente das carnes vermelhas, tem sido atualmente apontado como um fator de risco para o desenvolvimento de doenças crônicas ${ }^{1,28,29}$. Nesta pesquisa, esse comportamento esteve presente na alimentação de mais da metade dos entrevistados. Mais recentemente, levantamento do Vigitel ${ }^{12}$ apontou que em Porto Alegre/RS o consumo de carnes com excesso de gordura foi de 34,8\% $\left(\mathrm{IC}_{95 \%}\right.$ 31,9 - 37,8), e no restante dos estados, essa taxa variou de 24,5\% (Salvador/BA) a 46,1\% (Campo Grande/MS). Em outros anos, 2006, 2007 e 2008, no RS, o Vigitel observou prevalências de 40,4\%, $33,3 \%$ e $34,6 \%$, respectivamente ${ }^{11,30,31}$, porém, apesar deste decréscimo esse comportamento alimentar de risco ainda é elevado entre os gaúchos. O fato de Pelotas (RS) apresentar prevalências mais altas em relação a todos os inquéritos do Vigitel pode ser justificado por este inquérito realizar entrevistas apenas em domicílios de capitais brasileiras com linhas de telefonia fixa, e, devido a isso, entrevistar pessoas de maior escolaridade e poder aquisitivo. Ao contrário, o presente estudo identificou uma parcela expressiva de pessoas com nível econômico médio e baixo. Percebe-se que o consumo de carnes com excesso de gordura é predominante entre os homens e indivíduos com menos escolaridade, o que pode ser explicado pelo menor grau de esclarecimento sobre hábitos alimentares saudáveis, ou até mesmo pela falta de incentivo de órgãos públicos de saúde, quanto à adoção de atitudes de vida mais saudáveis na cidade.

As carnes menos consumidas neste estudo foram as vísceras, sendo que mais de 70\% dos consumidores referiram consumi-las menos de uma vez por semana. Essas carnes são ricas em ferro, vitaminas A e $\mathrm{D}^{2}$, e segundo o $\mathrm{MS}^{14}$, devem estar presentes na alimentação uma vez por semana, porém sem ultrapassar essa indicação pelo alto teor de colesterol. O consumo de uma vez por semana neste trabalho foi observado em menos de $10 \%$ dos entrevistados. O baixo consumo desta carne pode estar ligado à preferência alimentar e/ ou falta de hábito da população.

Como este estudo, foi amostrado por conglomerados, foi positivo o fato das análises terem levado em consideração o efeito deste processo amostral nos resultados, o que imprime maior precisão aos dados apresentados ${ }^{32}$. O consumo de peixes apresentou o maior DEF na amostra, indicando uma homogeneidade no consumo desta carne dentro de alguns conglomerados e uma heterogeneidade deste consumo entre os conglomerados. $\mathrm{O}$ consumo de frango apresentou o menor DEF, significando que a amostragem por conglomerados não influenciou este resultado, visto que, como já foi descrito na metodologia deste estudo, os conglomerados acabam se distinguindo em relação ao nível econômico de seus moradores, que costuma ser muito parecido.

\section{Conclusões}

As carnes são consumidas pela grande maioria da população, apesar de menos de $1 / 3$ dos entrevistados atenderem a um consumo diário. Preocupante foi a baixa frequência semanal do consumo de peixes, considerado saudável por seu conteúdo nutricional, paralelamente ao frequente consumo semanal da carne mais perigosa para a saúde, os embutidos, além da elevada prevalência de consumo de carnes com excesso de gordura.

Estes achados evidenciam a necessidade da criação de políticas públicas de saúde atuando 
efetivamente no incentivo a práticas mais saudáveis de alimentação estimulando e possibilitando o consumo mais frequente de carnes brancas, especialmente peixes, e também a correção de hábitos impróprios que elevam o risco de morbidades e diminuem a longevidade, promovendo o desestímulo do consumo de embutidos e de carnes com excesso de gordura.

\section{Colaboradores}

BC Schneider, SMS Duro e MCF Assunção participaram igualmente de todas as etapas de elaboração do artigo.

\section{Referências}

1. World Health Organization (WHO). The World Health Report 2002: Reducing Risks, Promoting Healthy Life. Geneva: WHO; 2002.

2. Bender A. Meat and meat products in human nutrition in developing countries. FAO Food Nutr Pap 1992; 53:1-91.

3. Hooper L, Thompson RL, Harrison RA, Summerbell CD, Ness AR, Moore HJ, Worthington HV, Durrington PN, Higgins JP, Capps NE, Riemersma RA, Ebrahim SB, Davey Smith G. Risks and benefits of omega 3 fats for mortality, cardiovascular disease, and cancer: systematic review. BMJ 2006: 332(7544):752-760.

4. Sidhu KS. Health benefits and potential risks related to consumption of fish or fish oil. Regul Toxicol Pharmacol 2003; 38(3):336-344.

5. Levy-Costa RB, Sichieri R, Pontes NS, Monteiro CA. Disponibilidade domiciliar de alimentos no Brasil: distribuição e evolução (1974-2003). Rev Saude Publica 2005; 39(4):530-540.

6. Instituto Brasileiro de Geografia e Estatística (IBGE). Pesquisa de Orçamentos Familiares 2008-2009: Despesas, Rendimentos e Condições de Vida. Rio de Janeiro: IBGE; 2010.

7. Instituto Brasileiro de Geografia e Estatística (IBGE). Pesquisa de Orçamentos Familiares 2002-2003: Análise da disponibilidade domiciliar de alimentos e do estado nutricional no Brasil. Rio de Janeiro: IBGE; 2004.

8. Cross AJ, Leitzmann MF, Gail MH, Hollenbeck AR, Schatzkin A, Sinha R. A Prospective Study of Red and Processed Meat Intake in Relation to Cancer Risk. PLoS Medicine 2007; 4(12):1973-1984.

9. Santarelli RL, Pierre F, Corpet DE. Processed meat and colorectal cancer: a review of epidemiologic and experimental evidence. Nutr Cancer 2008; 60(2):131-144.

10. Instituto Nacional do Câncer (INCA). Fatores de Risco para o Câncer - Hábitos Alimentares. 2010. [acessado 2010 out 7]. Disponível em: http://www1.inca.gov.br/ conteudo_view.asp?ID $=18$

11. Iser BPM, Yokota RTC, Sá NNB, Moura L, Malta DC Prevalência de fatores de risco e proteção para doenças crônicas nas capitais do Brasil - principais resultados do Vigitel 2010. Cien Saude Colet 2012; 17(9):2343-2356. 
12. Brasil. Ministério da Saúde (MS). Secretaria de Vigilância em Saúde. Vigitel Brasil 2010: Vigilância de Fatores de Risco para Doenças Crônicas por Inquérito Telefônico. Brasília: MS; 2011.

13. World Health Organization (WHO). Process for a Global Strategy on Diet, Physical Activity and Health. Geneva: WHO; 2003.

14. Brasil. Ministério da Saúde (MS). Secretaria de Atenção à Saúde. Coordenação-Geral da Política de Alimentação e Nutrição. Guia alimentar para a população brasileira: promovendo a alimentação saudável. Brasília: MS; 2006.

15. Prefeitura de Pelotas. Agro Indústria e Comércio. 2002 [acessado 2009 set 1]. Disponível em: http://www.pelotas.com.br/cidade_dados/pelotas_dados.htm

16. Instituto Brasileiro de Geografia e Estatística (IBGE). Censo Demográfico 2000. [acessado 2010 out 7]. Disponível em: www.ibge.gov.br/censo2000

17. Barros AJD, Menezes AMB, Santos IS, Assunção MCF, Gigante D, Fassa AG, Marques M, Araújo C, Hallal PC, Facchini LA. O Mestrado do Programa de Pós graduação em Epidemiologia da UFPel baseado em consórcio de pesquisa: uma experiência inovadora. Rev Bras Epidemiol 2008; 1(Supl. 11):133-144.

18. Associação Brasileira de Empresas de Pesquisa (ABEP). Critério de Classificação Econômica Brasil. São Paulo: ABEP; 2010.

19. Delgado CL. Rising Consumption of Meat and Milk in Developing Countries Has Created a New Food Revolution. American Society for Nutritional Sciences 2003; 133(Supl. 11):3907S-3910S.

20. Food and Agriculture Organization (FAO). Animal Production and Health Division. Livestock's Long Shadow: Environmental Issues and Options - LEAD. Roma: FAO; 2006.

21. Brasil. Ministério da Agricultura (MA). Estatísticas em Pecuária - Brasil: produção de carne suína, ovina e de frango. 2010. [acessado 2010 out 7]. Disponível em: http://www.agricultura.gov.br/

22. Marinho MCS, Hamann EM, Lima ACCF. Práticas e mudanças no comportamento alimentar na população de Brasília, Distrito Federal, Brasil. Rev Brasileira de Saúde Materno Infantil 2007; 7(3):251-261.
23. Fonseca MJM, Chor D, Valente JG. Hábitos alimentares entre funcionários de banco estatal: padrão de consumo alimentar. Cad Saude Publica 1999; 15(1):29-39.

24. Slattery ML, Jacobs Juniorr DR, Hilner JE, Caan BJ, Van Horn L, Bragg C, Manolio TA, Kushi LH, Liu KA. Meat consumption and its associations with other diet and health factors in young adults: the CARDIA study. Am J Clin Nutr 1991; 54(5):930-935.

25. Cosgrove M, Flynn A, Kiely M. Consumption of red meat, white meat and processed meat in Irish adults in relation to dietary quality. BrJ Nutr 2005; 93(6):933-942.

26. Mondini L, Monteiro CA. Mudanças no padrão de alimentação da população urbana brasileira (1962-1988). Rev Saude Publica 1994; 28(6):433-439.

27. Chan EJ, Cho L. What can we expect from omega- 3 fatty acids? Cleve Clin J Med 2009; 76(4):7.

28. Neves FJ, Koifman RJ, Mattos IE. Mortalidade por câncer de cólon e reto e consumo alimentar em capitais brasileiras selecionadas. Rev Brasileira de Epidemiologia 2006; 9(11):112-120.

29. Sarturi JB, Neves J, Peres KG. Obesidade em adultos: estudo de base populacional num município de pequeno porte no sul do Brasil em 2005. Cien Saude Colet 2010; 15(1):105-113.

30. Brasil. Ministério da Saúde (MS). Vigitel Brasil 2007: Vigilância de Fatores de Risco e Proteção para Doenças Crônicas por Inquérito Telefônico. Brasília: Secretaria de Vigilância em Saúde; 2008.

31. Brasil. Ministério da Saúde (MS). Vigitel Brasil 2008: Vigilância de Fatores de Risco e Proteção para Doenças Crônicas por Inquérito Telefônico. Brasília: Secretaria de Vigilância em Saúde; 2009.

32. Silva NN. Amostragem Probabilística. 2a ed. São Paulo: Edusp; 2004.

Artigo apresentado em 15/07/2013

Aprovado em 03/09/2013

Versão final apresentada em 11/09/2013 